\title{
Membrane proteins: functional and structural studies using reconstituted proteoliposomes and 2-D crystals
}

\begin{abstract}
Correspondence

J.-L. Rigaud

Institut Curie

UMR-CNRS 168 and LRC-CEA 8

11 Rue Pierre et Marie Curie, 75231

Paris, Cedex 05

France

E-mail: rigaud@ curie.fr

Presented at the XXX Annual Meeting of the Brazilian Society of Biochemistry and Molecular Biology, Caxambu, MG, Brazil, May 19-22, 2001

Research supported in part by CNRS (France)-CNPq (Brazil)

collaborative programs.

Received January 14, 2002

Accepted May 7, 2002

Reconstitution of membrane proteins into lipid bilayers is a powerful tool to analyze functional as well as structural areas of membrane protein research. First, the proper incorporation of a purified membrane protein into closed lipid vesicles, to produce proteoliposomes, allows the investigation of transport and/or catalytic properties of any membrane protein without interference by other membrane components. Second, the incorporation of a large amount of membrane proteins into lipid bilayers to grow crystals confined to two dimensions has recently opened a new way to solve their structure at high resolution using electron crystallography. However, reconstitution of membrane proteins into functional proteoliposomes or 2-D crystallization has been an empirical domain, which has been viewed for a long time more like "black magic" than science. Nevertheless, in the last ten years, important progress has been made in acquiring knowledge of lipid-protein-detergent interactions and has permitted to build upon a set of basic principles that has limited the empirical approach of reconstitution experiments. Reconstitution strategies have been improved and new strategies have been developed, facilitating the success rate of proteoliposome formation and 2-D crystallization. This review deals with the various strategies available to obtain proteoliposomes and 2-D crystals from detergent-solubilized proteins. It gives an overview of the methods that have been applied, which may be of help for reconstituting more proteins into lipid bilayers in a form suitable for functional studies at the molecular level and for high-resolution structural analysis.
\end{abstract}

\section{Introduction}

Screening of the genomes of different organisms has demonstrated that about $25 \%$ of the sequenced genes code for strongly hydrophobic proteins, which are integrated into cell membranes (1). These results have emphasized the importance of membrane proteins in many biological processes essential for life. However, the complexity of most

\section{Key words}

- Membrane proteins

- Proteoliposomes

- 2-D crystals

- Electron crystallography

- Reconstitution biological membranes makes it difficult to study these membrane proteins in situ. Therefore, purification from the native membrane and further reincorporation of the purified membrane protein into an artificial membrane to form proteoliposomes or to produce two-dimensional (2-D) crystals continue to be a crucial step in studying the function and structure of these molecules.

First of all, membrane protein reconstitu- 
tion into liposomes has played an important role and should continue to be a powerful tool that can be used to identify and characterize the mechanisms of action of membrane proteins. The necessity for reconstitution is due to the fact that many membrane proteins express their full activity only when correctly oriented and inserted into a lipid bilayer. In particular, reconstitution has played a central role in the identification and characterization of the mechanisms of action of membrane proteins with a vectorial transport function. More generally, biochemical and biophysical approaches have provided important information about lipid-protein and protein-protein interactions as well as topological and topographic features of different classes of membrane proteins reconstituted into proteoliposomes (2-5).

Second, structure determination at high resolution is actually a difficult challenge for membrane proteins and the number of membrane proteins that have been crystallized in three dimensions (3-D) is still small and far behind that of soluble proteins (6). Reconstitution of membrane proteins into artificial membranes to form 2-D crystals has opened a new way to solve their structures inside a native-like environment (7-10). Indeed, electron crystallography of 2-D crystals has developed to the point that significant structural atomic models have been built and that many membrane protein structures have been evaluated at resolutions allowing the secondary structure to be assessed (11). These recent results have made electron crystallography an excellent alternative and a complementary discipline to X-ray crystallography in membrane protein structural biology.

However, the ability to investigate functional aspects or to solve the structures of membrane proteins through the use of reconstituted systems has been limited by the fact that methods for producing high quality proteoliposomes or 2-D crystals have not advanced at the same rate as biochemical, biophysical and molecular biology techniques, on the one hand, and electron crystallography on the other. Thus, one of the limiting factors in obtaining molecular information was related to the lack of reproducible methods of reconstitution. Therefore enormous efforts were required to understand the mechanisms of reconstitution and for new approaches to be evaluated, refined and applied to available proteins in order to make reconstitution even more important as a tool for establishing structure-function relationships at the molecular level.

This review will deal with the different strategies commonly used to reconstitute proteoliposomes and 2-D crystals. It will also focus on recent new approaches, which have led to promising perspectives for the production of highly functional proteoliposomes and more 2-D crystals of membrane proteins amenable to structural analysis. General guidelines and rules will be proposed in this field which has long been seen more as art than as science.

\section{Reconstitution of proteoliposomes}

\section{Strategies}

From the analysis of the abundant literature concerning the insertion of membrane proteins into liposomes, four basic strategies have been outlined: mechanical means, freeze-thaw, organic solvents, and detergents. Although these reconstitution strategies have proven to be very useful to prepare pure phospholipid vesicles (12), the additional insertion of a membrane protein during the reconstitution process has imposed many constraints that have seriously hampered the applicability of phospholipid vesicles to proteoliposome reconstitution. Indeed, besides the need for conditions that preserve protein activity, many criteria have to be considered to fully optimize the reconstitution of a membrane protein: the homogeneity of protein insertion, the final orientation of the protein, the morphology and the size of the reconsti- 
tuted proteoliposomes, as well as their residual permeability. Even more limiting is the fact that many methods for preparing pure liposomes have been difficult to apply successfully to proteoliposome reconstitution because most membrane proteins are purified by the use of detergents, which interfere with the process of vesicle formation.

Thus, the vast majority of membrane protein reconstitution procedures are those involving the use of detergents. Indeed, due to their amphiphilic character, most membrane proteins require detergents, not only as a means of disintegrating the structure of native membranes in the initial step of their solubilization, but also as a means of keeping the protein in a non-denaturing environment during further purification (13). The standard procedure used to reconstitute a purified membrane protein involves comicellization of the protein in an excess of phospholipid and appropriate detergent, to form a solution of mixed lipid-protein-detergent and lipid-detergent micelles. Next, the detergent is removed from the micellar solutions, resulting in the progressive formation of closed lipid bilayers into which the proteins eventually incorporate. All the detergent-mediated reconstitutions described in the literature rely on this standard procedure but differ essentially in the techniques used to remove the detergent, which can be dialysis, gel chromatography, dilution, or hydrophobic adsorption onto polystyrene beads (Figure 1).

As shown by the abundant literature, reconstitutions from detergent micellar mixtures have yielded proteoliposomes of different sizes and compositions depending on the nature of the detergent, the particular procedure to remove it, as well as the nature of the protein and the lipid composition. Therefore, each membrane protein responded differently to the various reconstitution procedures and the approach has long been entirely empirical. In the 90 's, important knowledge of the mechanisms of liposome formation as well as understanding of the physical behavior of lipid-detergent systems permitted the construction of a set of basic principles that has limited the number of experimental variables and thus the empirical approach to proteoliposome reconstitution $(5,14,15)$.

To allow realistic monitoring of the mechanisms by which proteins may associate with lipids during detergent-mediated reconstitutions, we have developed a strategy (Figure 2) based on the idea that the process of reconstitution by detergent removal is the reverse of the process of solubilization by increasing amounts of detergent $(5,16)$. To this end, the first stage in our strategy was to add detergent to preformed liposomes using the entire range of detergent concentrations that cause the transformation of lamellar structures to mixed micelles (1719). The lipid-detergent mixed amphiphilic structures that are formed during the solubilization process are similar to those pre-

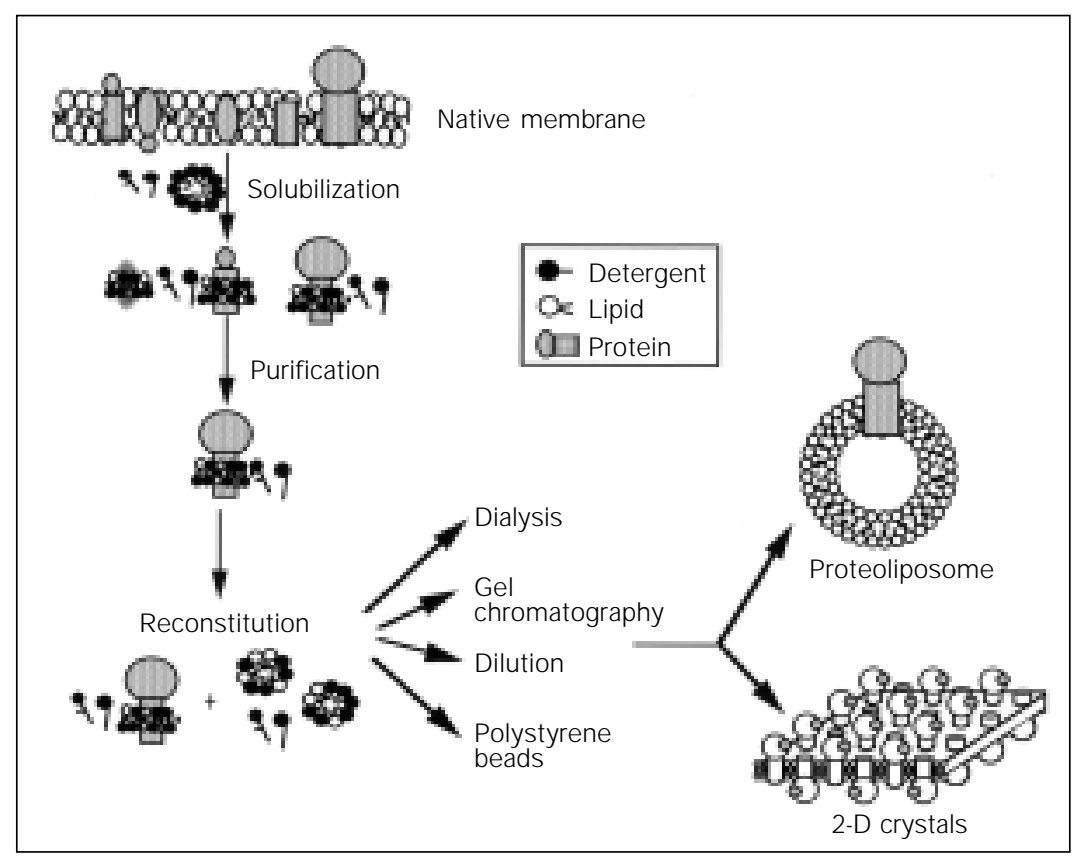

Figure 1. Detergent-mediated reconstitutions. Most membrane proteins are extracted from native membranes with solubilizing detergent concentrations. After purification, the solubilized protein is supplemented with an excess of lipids and detergents, leading to a solution of mixed lipid-protein-detergent and lipid-detergent micelles. For proteoliposome or 2-D crystal reconstitution, detergent is removed from these micellar solutions using different strategies. 
dicted to form during the micelle-to-lipid bilayer transition in a reconstitution process by detergent removal. Thus, in a second stage, adding the protein at each step of the solubilization process allowed a "snap-shot" of all the situations that might occur in a reconstitution experiment following detergent removal from lipid-protein-detergent micelles. Finally, in a third stage, the deter-

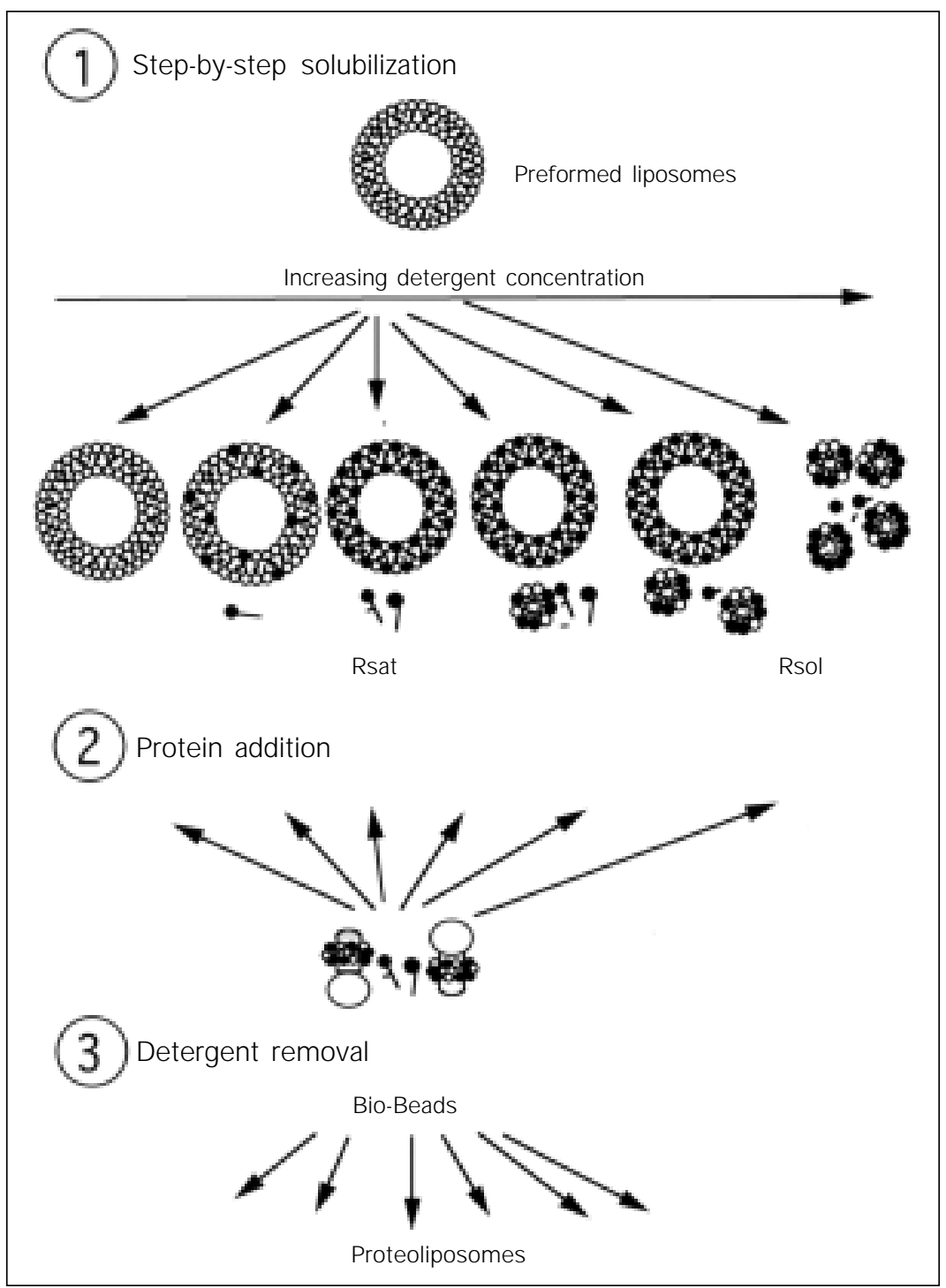

Figure 2. The "step-by-step" reconstitution strategy. The standard procedure for reconstituting membrane proteins into proteoliposomes consists of three stages. 1, Step-by-step addition of detergent to preformed liposomes. A few steps in the solubilization process are schematically drawn. Rsat corresponds to the detergent-to-lipid ratio in liposomes at the onset of solubilization, while Rsol corresponds to the detergent-to-lipid ratio in micelles when total solubilization is reached. 2, Addition of the solubilized protein in each welldefined step of the solubilization process. 3, Detergent removal. gent was removed and the resulting proteoliposomes were characterized in terms of protein incorporation and orientation, vesicle size distribution, and biological activity.

With respect to optimal protein incorporation, we have identified three mechanisms by which membrane proteins can associate with lipids to give proteoliposomes (Figure 3). Depending on the nature of the detergent, proteins can be either directly incorporated into detergent-saturated liposomes (octylglucoside- and dodecylmaltoside-mediated reconstitutions), transferred from mixed micelles to detergent-saturated liposomes (Triton X-100-mediated reconstitutions) or participate in proteoliposome formation during the micellar-to-lamellar transition (cholate, chaps, and $\mathrm{C}_{12} \mathrm{E}_{8}$-mediated reconstitutions).

In addition to providing original information about the mechanisms of lipid-protein association in the presence of detergent, this "step-by-step" reconstitution strategy proved to be a powerful reconstitution procedure, more suitable than the usual methods. This new reconstitution strategy, which is now widely used, has produced proteoliposomes which sustain biological activities comparable to those measured in the native membrane. Indeed, it has allowed an easy determination of the optimal conditions under which a protein can associate with lipids in the presence of detergent. In particular, it should be stressed that reconstitution of a membrane protein into detergentsaturated preformed liposomes ensured a unidirectional insertion of the protein in the membrane and generally produced the most efficient proteoliposomes (20-22). An additional advantage of our new reconstitution strategy relied on the use of Bio-Beads SM2 to remove the detergent. Indeed, the efficient removal of detergent from reconstituted proteoliposomes was an absolute necessity since residual detergent might inhibit enzymatic activity and/or drastically increase the passive permeability of the liposomes, rendering transport functions difficult to measure. 
Systematic studies using radioactive detergents have demonstrated the efficiency of polystyrene beads to allow almost complete removal of any kind of detergent, making this method of detergent removal much more efficient than conventional dialysis $(23,24)$.

\section{Functional studies}

An important benefit of the production of "quasi-ideal" proteoliposomes has been to obtain original information about the function of different membrane proteins. A few notable examples from our laboratory include the demonstration of a back pressure effect of $\Delta \mathrm{pH}$ on bacteriorhodopsin (25), the evidence of a direct $\mathrm{Ca}^{2+} / \mathrm{H}^{+}$coupling in $\mathrm{Ca}$ ATPases (26-28), and the analysis of artificial $\Delta \mu \mathrm{H}^{+}$driving ATP synthesis by $\mathrm{F}_{0} \mathrm{~F}_{1}$ ATPases (21). It has also been possible to co-reconstitute two functionally coupled membrane proteins, permitting the analysis of coupling $\Delta \mu \mathrm{H}^{+} / \mathrm{ATP}$ synthesis in co-reconstituted systems containing $\mathrm{F}_{0} \mathrm{~F}_{1}$ ATPases and different light-driven proton generators (29-32).

More recently, using reconstituted proteoliposomes, we have been able to mimic and analyze at the molecular level the mechanisms of transfer of the phage genome into bacteria during phage infection. In Escherichia coli, the mere interaction of phage $\mathrm{T} 5$ with FhuA, its outer membrane receptor, is sufficient to trigger the release of DNA from the phage capsid. Therefore, by reconstituting the membrane receptor FhuA into liposomes, we have been able to analyze the mechanism of phage infection, namely phage binding and the subsequent transport of its DNA into the proteoliposomes (Figure 4).

The transfer of DNA has been visualized using cryo-electron microscopy, i.e., samples frozen in vitreous ice (33). When T5 phages were added to FhuA-containing proteoliposomes, electron micrographs clearly demonstrated that phages were able to bind to their receptor incorporated in the membranes of proteoliposomes (Figure 4A-C). Many capsids of phages bound to their receptor were partially or totally devoid of DNA, and, interestingly, always associated with proteoliposomes that contained DNA, providing clear evidence of DNA transfer from the phage

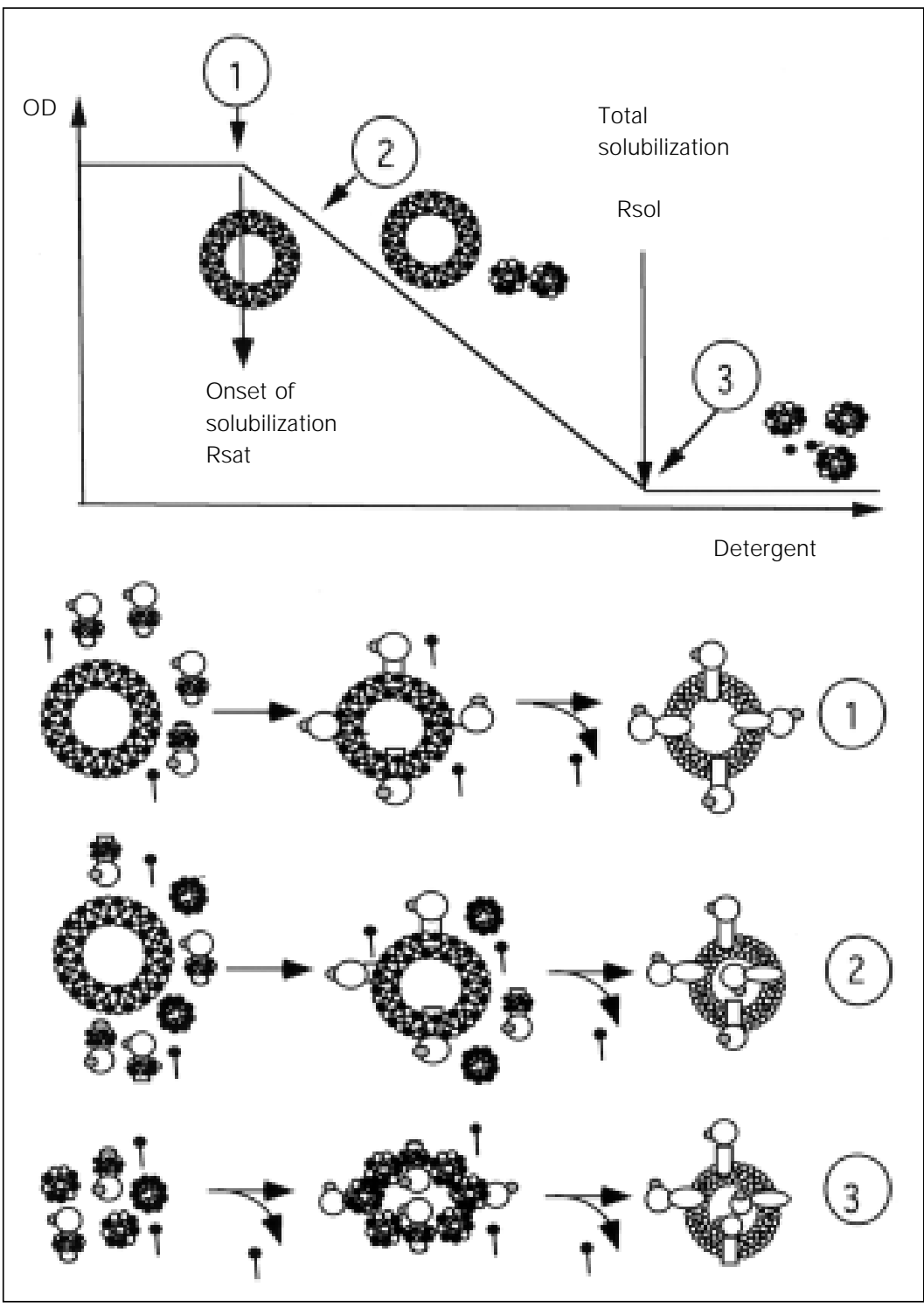

Figure 3. Mechanisms of protein incorporation in detergent-mediated reconstitutions. Proteoliposomes were reconstituted by the "step-by-step" strategy described in Figure 2. The upper part of the Figure describes the steps in the lamellar-to-micellar transition when optimal reconstitution was observed. The lamellar-to-micellar transition can be analyzed by turbidimetry as schematically shown. Depending upon the nature of the detergent, three mechanisms have been identified: direct protein incorporation into detergent-saturated liposomes at the onset of solubilization (Rsat, mechanism 1), transfer from mixed micelles to detergent-saturated liposomes (Rsol, mechanism 2) or proteoliposome formation by micellar coalescence (mechanism 3). Note that the final orientation of the protein depends on the mechanism of association. 
capsid into the proteoliposomes. More than one complete genome $(121,000 \mathrm{bp}, 40 \mu \mathrm{m}$ in length) could be transferred into proteoliposomes of $150 \mathrm{~nm}$ in diameter and several genomes were observed in proteoliposomes of $250 \mathrm{~nm}$ in diameter (Figure 4A).

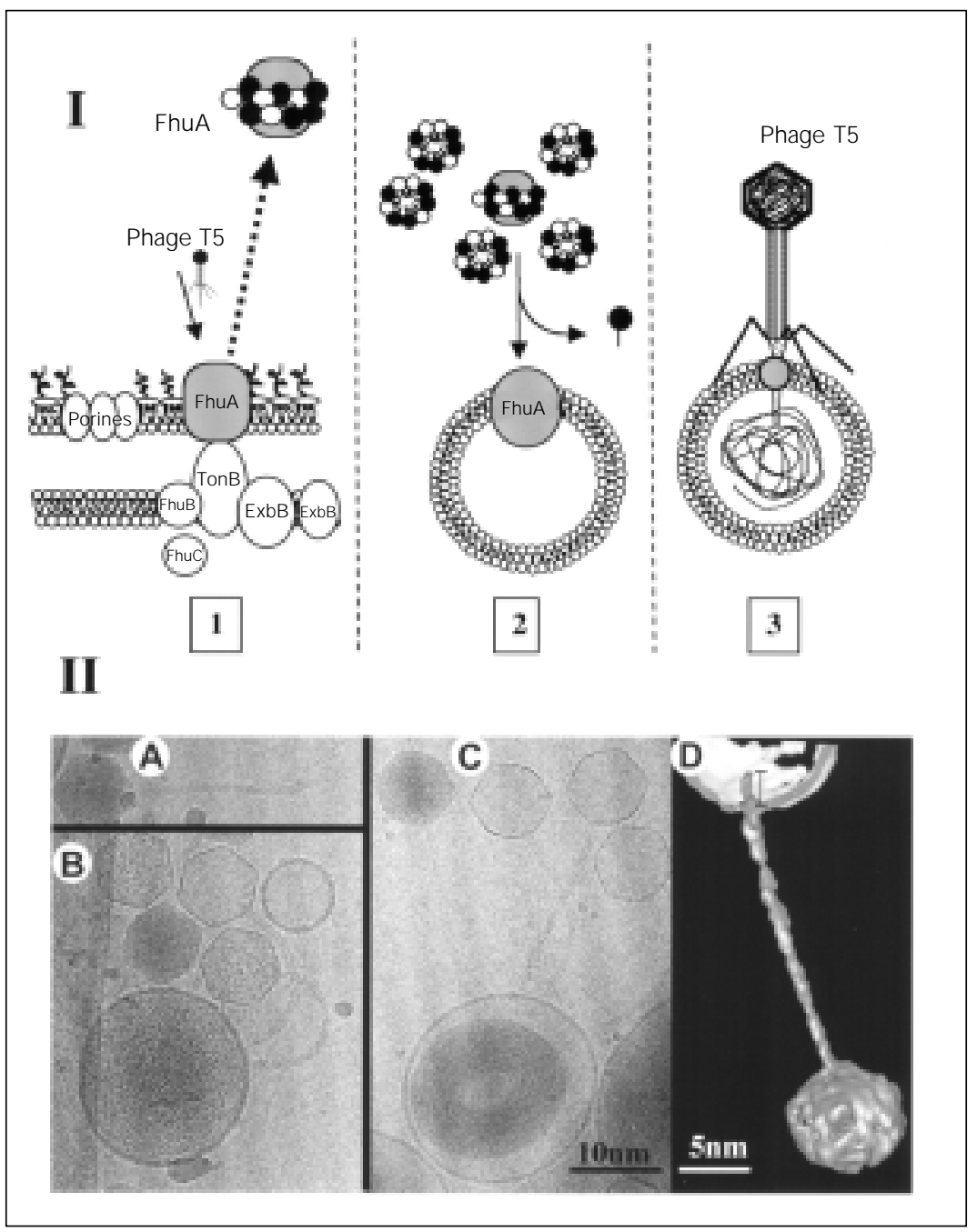

Figure 4. Use of FhuA-containing proteoliposomes to study the mechanism of phage infection. I, The proteoliposome strategy. 1, Purification of FhuA, the receptor for phage T5 on the outer membrane of Escherichia coli. 2, Reconstitution of FhuA into proteoliposomes. 3, Binding of T5 and DNA release into proteoliposomes. II, Panels A, B, and C illustrate cryoelectron micrographs demonstrating the DNA transfer from phage T5 (A) into FhuA-containing proteoliposomes in the absence (B) or presence of $50 \mathrm{mM}$ spermine (C). DNA is visualized in electron-dense material either in full and partially empty icosahedral capsids or inside proteoliposomes in which DNA transfer has occurred. Note that, as opposed to what is observed in the absence of spermine, DNA strands transferred into spermine-containing proteoliposomes appear to be condensed into a single toroidal structure. Panel D, Isosurface representation of the 3-D reconstruction of the phage bound to the proteoliposome as determined by electron tomography. The top of the vesicle has been cropped to reveal the tip of the phage visible inside the vesicle.
For a better understanding of the interaction of the phage with its receptors, information has been retrieved from 3-D images of phage-proteoliposome complexes using electron tomography (34). The principle of electron tomography consists of the determination of the 3-D structure of a single object from a series of 2-D images of this object tilted at various angles (35). The resolution of the 3-D reconstructions demonstrated unambiguously that, after binding to its receptor, the straight fiber of the phage crossed the lipid bilayer and underwent a major conformational change, allowing the transfer of its double-stranded DNA into the proteoliposome (Figure 4C). On the basis of the crystal structure of the FhuA receptor (36), we have been able to conclude that FhuA was only used as a docking site for the phage and that the tip of the phage tail acted like a DNA "injection needle" creating a passageway at the periphery of FhuA.

Besides the important consequences of our study for the molecular mechanisms of phage infection, the experimental approach using reconstituted proteoliposomes has important applications in many biological contexts. For example, it has been an attractive model system to study the mechanisms of DNA condensation. To this end, proteoliposomes were reconstituted in the presence of different amounts of spermine, a well-known DNA condensing agent. In the presence of $50 \mathrm{mM}$ spermine-containing liposomes, the DNA strands transferred from the phage capsids appeared condensed into characteristic toroidal structures that occupied a volume smaller than the internal volume of the proteoliposome (Figure 4B). Increasing the number of DNA strands transferred into the proteoliposomes did not change the number of toroids in the liposomes but increased significantly the size of the toroid formed (37). From another point of view, the approach could be important for gene therapy applications, since the DNA-containing proteoliposomes produced in this way could 
serve as alternative vehicles for the transfer of foreign genomes into eukaryotic cells.

\section{Reconstitution of 2-D crystals}

Elucidation of the structure of membrane proteins is a major challenge. Indeed, less than 30 original structures of membrane proteins have been solved to atomic resolution, a number which lags far behind that of soluble proteins, with several thousand accurately determined structures.

While conventional 3-D crystallization has provided most of the atomic structures of membrane proteins, successful growth of 3 -D crystals of membrane proteins in detergent micelles is relatively infrequent. This drawback is mainly related to the difficulty in maintaining a crystal lattice through the sole interactions between the hydrophilic domains of the proteins, with the hydrophobic domains being shielded by the detergent micelles. To help with this problem, extensive studies have been performed to develop new strategies. In particular, a novel approach has been introduced by Michel's group (38), in which monoclonal antibody fragments were specifically bound to proteins, increasing the interactions between the hydrophilic domains of membrane proteins. More recently, bi-continuous lipidic cubic phases have been successfully used as matrices for nucleation and further 3-D crystallization of different membrane proteins, with the idea that proteins in such membrane mimetic systems should be more stable than in micelles (39).

It is noteworthy, that reconstitution of membrane proteins into artificial membranes to form crystals confined to two dimensions has opened a new way to solve their structure (7-10). In several cases, the structures obtained by electron crystallography allowed atomic models to be built. Besides these examples, many other 2-D crystals have been analyzed at resolution which allowed the secondary structure to be clearly solved. Such knowledge, combined with the information from sequence analysis, frequently provided significant insight into the structure of the protein. However, as for 3-D crystallization, the production of ordered 2-D crystals remains rare and many proteins continue to resist efforts. Except for few examples of 2$D$ crystals of membrane proteins in native membranes, 2-D crystals have to be reconstituted from solubilized purified proteins.

\section{2-D crystallization by detergent removal in bulk solution}

To date, the most frequently employed strategy for 2-D crystallization relies on the general method of detergent-mediated reconstitution of proteoliposomes, but at low lipid/protein ratios (1 protein molecule per 10-50 lipid molecules for 2-D crystals as compared to 1 protein molecule per 200010,000 lipid molecules for proteoliposomes). The strategy consists of starting with the purified protein and a suitable combination of lipids, both solubilized in detergent. Next, the detergent is removed from these micellar solutions, resulting in the progressive formation of lipid bilayers into which the proteins incorporate and eventually crystallize. Several types of 2-D crystals, all containing a continuous lipid bilayer in which proteins have been incorporated, can be produced: vesicular crystals, tubular crystals in which reconstituted proteins are helically ordered on the surface of a cylinder, and planar crystalline sheets which in some instances can lead to thin 3-D crystals upon the stacking of the 2-D arrays (Figure 5).

Different methods have been used to remove detergent, each presenting advantages and disadvantages (for a recent review, see Ref. 10). Dialysis is the most widely used technique in 2-D crystallization trials. Due to the necessity to scale down the amount of material, microdialysis devices have been used in the form of small compartments (50-100 $\mu \mathrm{l})$ dialyzed against large 
buffer volumes. The dialysis method has been successfully applied to many membrane proteins, but may not be well suited for detergents with low critical micellar concentrations, that require a long time for dialysis.

The method of detergent removal by hydrophobic adsorption onto polystyrene beads, previously shown to be very efficient for proteoliposome reconstitution (24), has been recently demonstrated to be a powerful alternative to conventional dialysis for 2-D crystallization trials (40). Using radioactive detergents, the method has been calibrated precisely in terms of the adsorptive capacity of the beads and the rates of detergent removal. The mechanisms underlying detergent adsorption onto beads have been analyzed and general rules for the use of polystyrene beads have been proposed. Sufficient reproducibility can be now obtained with experience and careful handling. An important benefit of this new strategy has been to produce new 2-D crystals of several membrane proteins

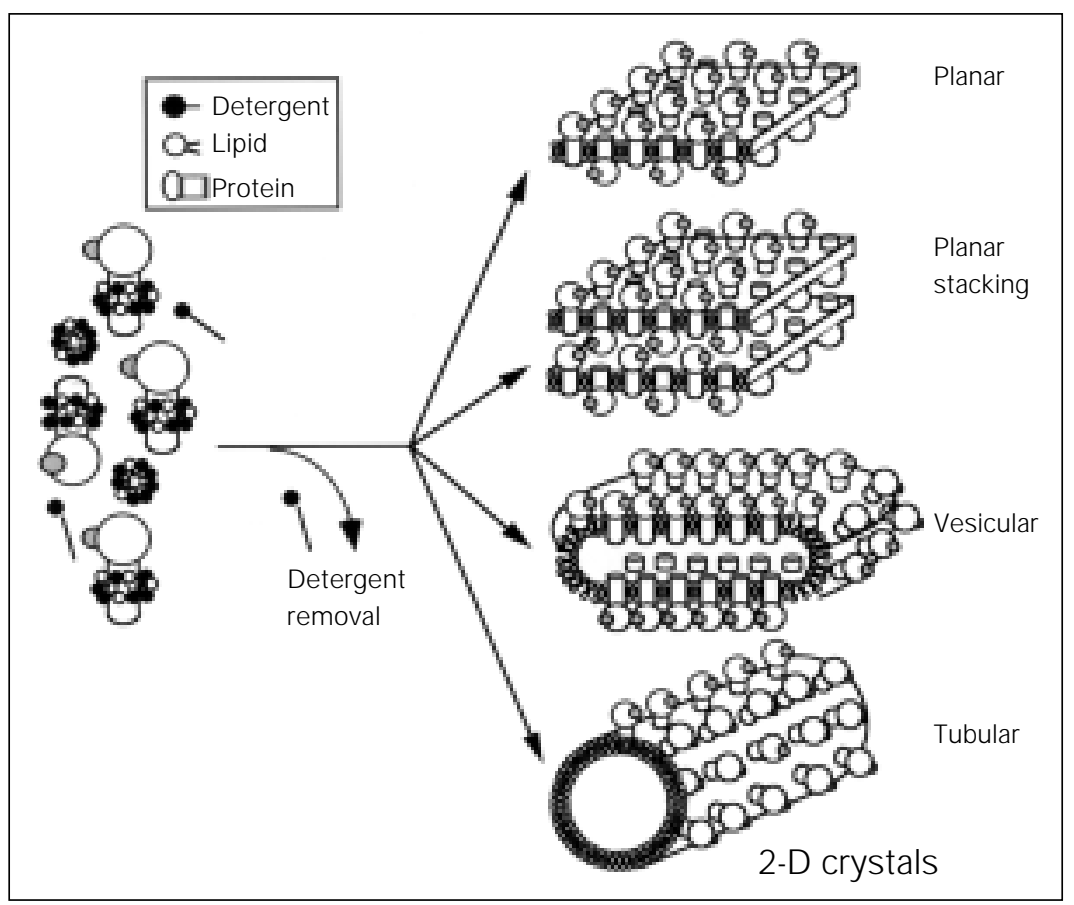

Figure 5. 2-D crystallization of membrane proteins by detergent removal. Following detergent removal from a lipid-protein-detergent micellar solution (lipid-to-protein ratios below 1 w/w), different 2-D crystals can be produced. solubilized in various detergents and, importantly, some of these 2-D crystals have been useful for high-resolution structural analysis $(36,41-43)$.

The self-assembling process leading to densely packed vesicles and 2-D crystals depends upon a large matrix of variables. In addition to the composition of the solution (salt, $\mathrm{pH}$, additives such as glycerol) and temperature, variables of primary importance are related to protein-protein, lipid-protein, detergent-protein and detergent-lipid interactions. As opposed to proteoliposome reconstitution, the rules for 2-D crystallization by detergent removal are far from being generalized and very few physicochemical parameters have been determined as essential for growing 2-D crystals of membrane proteins $(10,44,45)$.

\section{Induced 2-D crystallization}

The previous reconstitution procedure implies that, upon detergent removal, proteins must be incorporated into the bilayer but also find homogeneous optimal interactions at this transition. These two simultaneous processes appear crucial to the outcome of 2-D crystallization trials and may explain the difficulty for many proteins to crystallize. For those membrane proteins difficult to crystallize, one can consider bilayer formation separately from crystallization and improve the strategy of "induced 2-D crystallization". In this case, reconstitutions should be first performed at high lipid-to-protein ratios, leading to proteoliposomes with proteins homogeneously incorporated and rather closely packed. Then, in a second step, crystallization could be induced by physical treatment such as lipid temperature transition (46) or chemical agents including vanadate that cross-links P-type ATPases (47) or phospholipase A2 that decreases the lipid-to-protein ratio in the reconstituted proteoliposome (48).

We have developed this approach to in- 
duce 2-D crystallization of Ca-ATPase after reconstitution into proteoliposomes (47). CaATPase-dense proteoliposomes, produced through the "step-by-step" reconstitution strategy, were demonstrated to be ideal to induce further crystallization by the addition of vanadate, leading to very long ordered crystalline tubes. These tubular crystals were produced by very few preparations of native sarcoplasmic reticulum vesicles, and the unknown parameters that control the growth of long tubes from spherical vesicles could be easily analyzed in our reconstituted system. In particular, we found that a critical step to make extended tubular crystals from small proteoliposomes was the inclusion of specific lipids that destabilize bilayers.

\section{2-D crystallization of membrane proteins on functionalized lipid layers}

Considerable interest exists in developing new strategies to increase the success rate of 2-D crystallization. The technique of 2-D crystallization on lipid layers is based on a specific interaction between the protein and specific ligands coupled to lipid molecules incorporated into a planar lipid film at the air-water interface. This method has proven to be a successful approach to 2-D crystallization of soluble proteins, providing important structural information $(49,50)$. The question as to whether this method was also applicable to membrane proteins was a challenge.

As the result of a careful study, we have recently been able to produce on planar lipidic templates the first 2-D crystals of two radically different membrane proteins, FhuA and $\mathrm{F}_{0} \mathrm{~F}_{1}$ ATPase, demonstrating the feasibility of this new strategy for membrane proteins $(51,52)$. A model for the mechanisms of 2-D crystallization of membrane proteins on a lipid layer has been proposed, which include three steps (Figure 6): i) binding of protein-lipid-detergent micelles to the lipid layer. Since many membrane proteins are overproduced and purified using recombinant proteins containing a stretch of continuous histidine residues (His-tag), a nickelchelating lipid has been employed as a general adaptor molecule that will link any Histag protein; ii) reconstitution of a bilayer around the pre-bound proteins by detergent removal using polystyrene Bio-Beads in the solution below the lipid layer; iii) diffusion of the lipid-protein complexes and further 2D crystallization.

This innovative strategy opens a new promising field for membrane protein structure determination since: i) it may increase the chances of success to produce 2-D crystals of proteins difficult to crystallize by conventional methods; ii) it permits structure analysis with smaller amounts of material. Indeed, since this strategy induces protein concentration at the interface, this leads to the use of less than $1 \mu \mathrm{g}$ protein as compared to $25-50 \mu \mathrm{g}$ protein required by the classical bulk method. This will be of par-

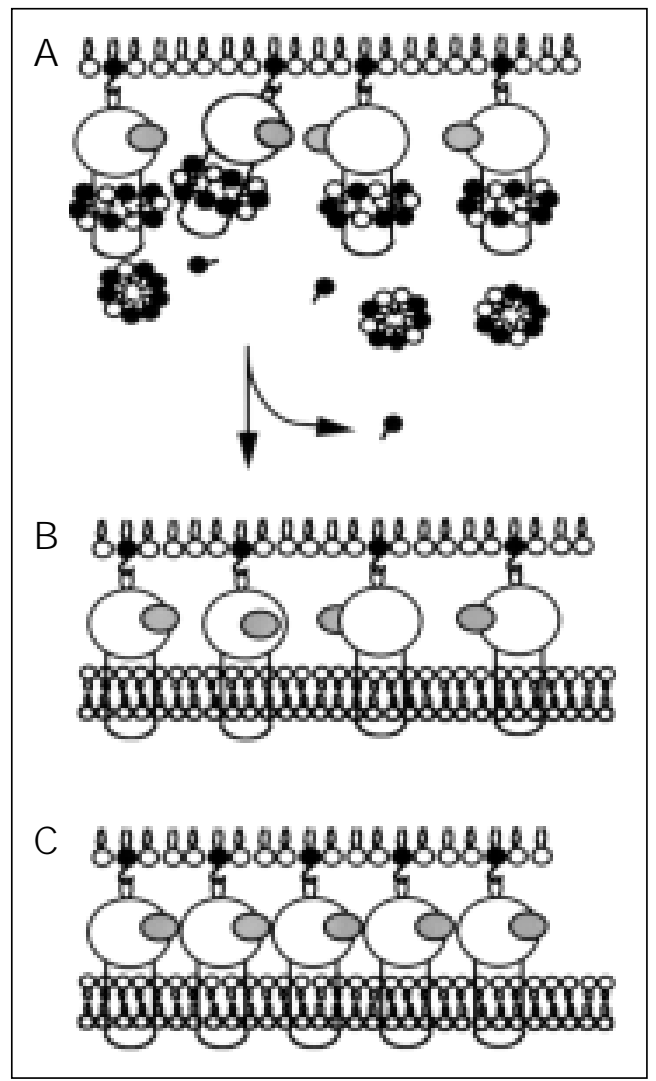

Figure 6. 2-D crystallization of membrane proteins on the lipid layer at the air-water interface. The three stages in the process of 2-D crystallization on lipid layers. A, Binding of protein-lipiddetergent micelles to the functionalized lipid layer spread at the air-water interface. B, Reconstitution of proteins into a lipid bilayer upon detergent removal. C, 2-D crystallization. 
ticular help to laboratories studying membrane proteins which are currently in the early stages of developing procedures for overexpression and large-scale production; iii) although a nickel-chelating lipid has been employed as a general adaptor that can link any His-tag protein at the surface, the strategy has been extended to other types of specific molecular recognition such as electrostatic interactions between proteins and lipids (52). Also it will be important to extend the strategy to other types of specific molecular recognition at the surface between proteins and lipids using other lipid-coupled physiological ligands.

\section{Structural analysis}

Electron crystallography. The method of choice for analyzing the results of 2-D crystallization is electron microscopy. The quickest specimen preparation is negative staining which yields excellent contrast but does not preserve high-resolution details. Once the optimal conditions of crystallization have been determined by negative staining, cryoelectron microscopy is preferred because it preserves the native structure of the protein to high resolution. One drop of the sample is deposited onto a carbon-coated grid, blotted with a filter paper and rapidly frozen in a liquid ethane bath (53). The frozen samples need to be maintained at $-170^{\circ} \mathrm{C}$ throughout observation under the microscope to preserve them against dehydration in the vacuum of the microscope and against radiation damage. Finally, the best method to assay the degree of order is electron diffraction, which is generally used to assess high-resolution structure analysis of good quality 2-D crystals.

Once the 2-D crystals have been produced, the structure of the protein can be determined by electron crystallography and image processing to a resolution that depends on the quality of the 2-D crystal (11). The best electron micrographs are selected by optical diffraction with a laser diffracto- meter, digitized on a microdensitometer and computer processed. The digitized images are Fourier transformed and phases and amplitudes of structure factors selected and averaged. In the course of image processing, crystal defects (lattice distortion) and image defects (lack of focus and astigmatism) are corrected. The inverse Fourier transform then generates a projection map which can be represented as a topographic map in which the hills would reflect higher electron densities. To determine the 3-D structure, electron diffraction patterns and electron images of 2-D crystals are recorded with the specimen at various tilt angles. By combining the structure factors which contribute to these projections in 3-D, a 3-D data set is compiled. The inverse transform of this data set is the 3-D map of the membrane protein.

Atomic force microscopy. As a complementary technique, atomic force microscopy (AFM) has become an important tool to investigate transmembrane proteins under physiological conditions. AFM measures the heights of biological samples to obtain topographic maps of protein structures protruding from the membrane at a lateral resolution of $\sim 8 \AA$ and a vertical resolution of $\sim 1 \AA$ (54). Specifically, high-resolution topographic maps before and after proteolytic cleavage of distinct protein domains have permitted the localization of these domains and the assignment of the sidedness of the whole transmembrane molecule. This approach was nicely illustrated by a recent study on the light-harvesting complex 2 (LH2) of Rubrivivax gelatinosus, the accessory antenna proteins in the bacterial photosynthetic apparatus built of $\alpha \beta$-heterodimers (55). AFM has been used to measure the surface topography of $\mathrm{LH} 2$ in a reconstituted membrane and to locate the C-terminal hydrophobic extension of 21-amino acid residues in the $\alpha$-subunit. High-resolution topographic maps revealed a nonameric organization of the $\alpha \beta$-heterodimers, regularly packed as cylindrical complexes incorpo- 
rated into the membrane in both orientations (Figure 7). Native LH2 showed one surface which protruded by $\sim 5 \AA$ and one which protruded by $\sim 14 \AA$ from the membrane. Topographic maps of samples reconstituted with thermolysin-digested LH2 have revealed a height reduction of the strongly protruding surface to $\sim 9 \AA$, while weakly protruding rings remained unchanged. These results demonstrated the extrinsic localization of the C-terminal domain of the $\alpha$-subunit of $R$. gelatinosus and allowed the periplasmic surface to be assigned.

Finally, the AFM has developed to the point that it permits to unfold membrane proteins, to detect flexible and stable extrinsic domains and to monitor function-related conformational changes by the addition of substrate molecules to the scanning buffer. Excellent reviews from Engel's group (5457) are available that illustrate various applications of AFM.

\section{Conclusions}

Reconstitution of membrane proteins into liposomes has played an important role and should continue to be a potentially powerful tool to be used to identify the mechanism of action of membrane proteins. As shown in this review, it appears that reconstitution no longer is "black magic" and the prospects of achieving optimal reconstitution of proteoliposomes are obviously good, with the use of

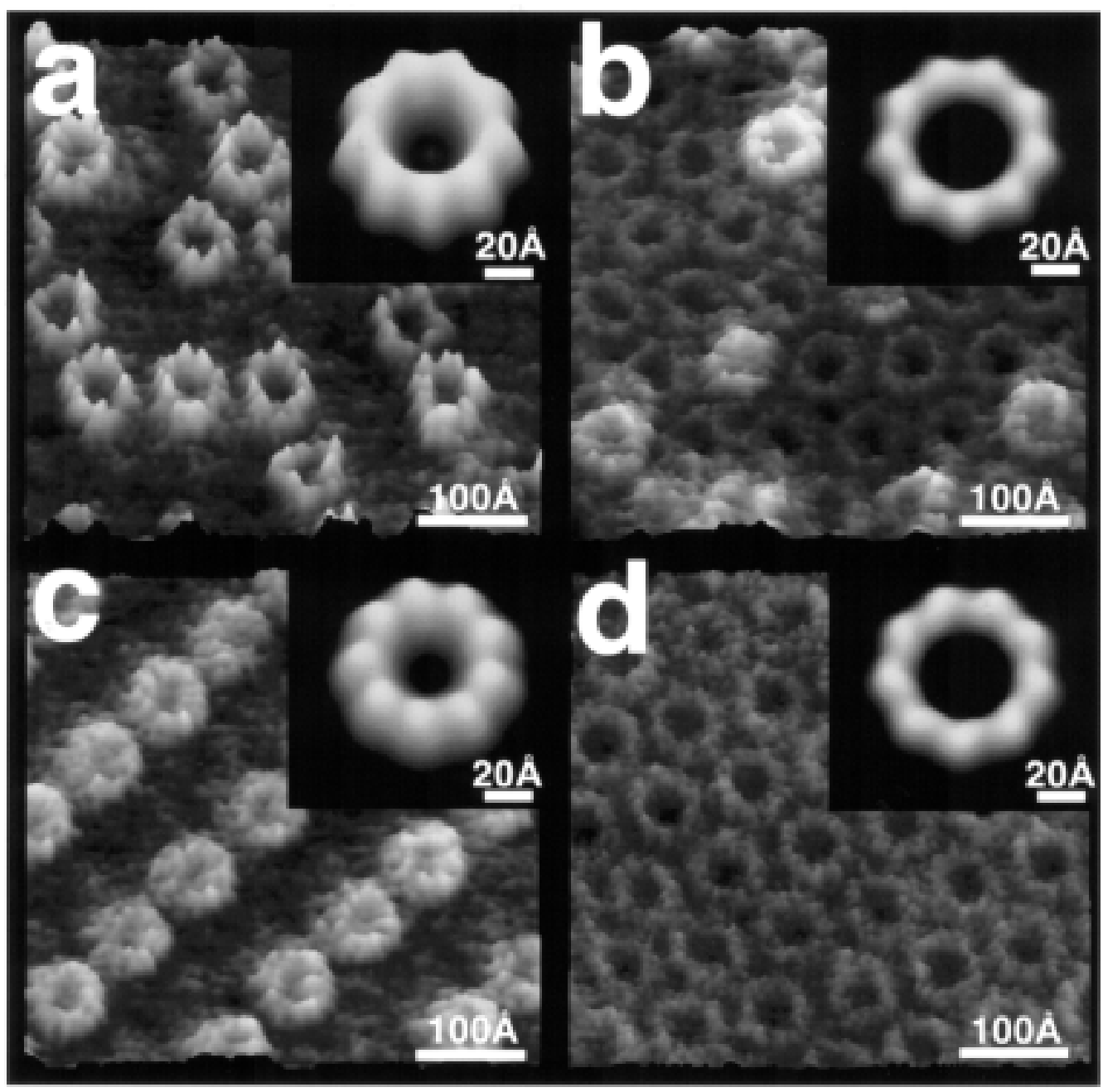

Figure 7. High-resolution atomic force microscopy topographic maps of the LH2 complex from Rubrivivax gelatinosus. a, Topographic maps of the strongly ( 14 Å) protruding surface of the native LH2. b, Topographic maps of the weakly $(\sim 5 \AA)$ protruding surface of the native LH2. C, Topographic maps of the strongly $(\sim 9 \AA)$ protruding surface of the digested $\mathrm{LH} 2$. d, Topographic maps of the weakly $(\sim 5 \AA)$ protruding surface of the digested LH2. Insets show average images of LH2 complexes calculated from the raw data shown in each corresponding panel. Proteolysis only influenced the topography of the strongly protruding surface, allowing to localize the C-terminal position of the $\alpha$-subunit. 
reliable methods.

Despite significant progress in the last few years, 3-D crystallization of membrane proteins has proved to be difficult and slow, and alternative approaches to structure determination have become essential. Electron crystallography, associated with 2-D crystallization, is a technique that has produced many structures and is definitely a viable alternative strategy to X-ray crystallography. However, for electron crystallography to become even more important, enormous efforts are required: i) for a deeper understanding of the 2-D crystallogenesis process, and ii) for the development of new approaches to be evaluated, refined and applied to available proteins.

The future of membrane protein reconstitution appears bright in the light of the steadily increasing number of membrane pro- teins, which have been identified in the sequencing of the genomes of different organisms. Powerful methods of gene overexpression are now available which will permit the production of new interesting membrane proteins.

\section{Acknowledgments}

I would like to thank Prof. A. Pereira and the Brazilian Society of Biochemistry and Molecular Biology for their kind invitation to attend the meeting in Caxambu, which gives me the great opportunity to establish contact with many laboratories in Brazil. Special thanks are due to D. Lévy, O. Lambert, M. Chami, S. Scheuring and B. Pitard who have been directly involved in many of the reconstitution studies described in this review.

\section{References}

1. J ones DT (1998). Do transmembrane protein superfolds exist? FEBS Letters, 423: 281-285.

2. Racker E (1982). Reconstitution of membrane processes. Methods in Enzymology, 55: 699-711.

3. Eytan GD (1981). Use of liposomes for reconstitution of biological functions. Biochimica et Biophysica Acta, 694: 185-202.

4. Comelius F (1991). Functional reconstitution of the sodium pump. Kinetics of exchange reactions performed by reconstituted Na/K-ATPase. Biochimica et Biophysica Acta, 1071: 19-66.

5. Rigaud J L, Pitard B \& Lévy D (1995). Reconstitution of membrane proteins into liposomes: application to energy-transducing membrane proteins. Biochimica et Biophysica Acta, 1231: 223-246.

6. Ostermeier C \& Michel H (1997). Crystallization of membrane proteins. Current Opinion in Structural Biology, 7: 697-701.

7. Kuhlbrandt W (1992). Two-dimensional crystallization of membrane proteins. Quarterly Reviews in Biophysics, 25: 149.

8. J ap BK, Zulauf M, Scheybani T, Hefti A, Baumeister W, Aebi U \& Engel A (1992). 2D crystallization: from art to science. Ultramicroscopy, 46: 45-84.

9. Hasler L, Heymann J B, Engel A, Kistler J
\& Walz T (1998). 2D crystallization of membrane proteins: rationales and examples. J ournal of Structural Biology, 121: 162-171.

10. Rigaud J L, Chami M, Lambert O, Lévy D $\&$ Ranck J (2000). Use of detergents in 2D crystallization of membrane proteins. Biochimica et Biophysica Acta, 1508: 112128.

11. Walz T\& Grigorieff N (1998). Electron crystallography of two-dimensional crystals of membrane proteins. J ournal of Structural Biology, 121: 142-161.

12. Szoka F \& Papahadjopoulos D (1980). Comparative properties and methods of preparation of lipid vesicles. Annual Review of Biophysics and Bioengineering, 9: 467-508.

13. Lemaire $M$, Champeil $P \&$ Moller J V (2000). Interaction of membrane proteins and lipids with solubilizing detergents. Biochimica et Biophysica Acta, 1508: 86111.

14. Lichtenberg D (1985). Characterization of the solubilization of lipid bilayers by surfactants. Biochimica et Biophysica Acta, 821: 470-478.

15. Silvius J R (1992). Solubilization and functional reconstitution of biomembrane components. Annual Review of Biophysics and Biomolecular Structure, 21: 323-
348.

16. Rigaud J -L \& Lévy D (2002). Reconstitution of membrane proteins into proteoliposomes. Methods in Enzymology: Liposomes, Part $B$ (in press).

17. Lévy $D$, Gulick $A$, Seigneuret $M \&$ Rigaud J -L (1990). Phospholipid vesicle solubilization and reconstitution by detergent. Symmetrical analysis of the two processes using octaethylene glycol mono-N-dodecyl ether. Biochemistry, 29: 9480-9488.

18. Paternostre $M T$, Roux $M \&$ \& Rigaud J $-L$ (1988). Mechanisms of membrane protein insertion into liposomes during reconstitution procedures involving the use of detergents. 1. Solubilization of large unilamellar liposomes (prepared by reverse-phase evaporation) by Triton X-100, octyl glucoside and sodium cholate. Biochemistry, 27: 2668-2677.

19. Lambert O, Lévy D, Ranck J L, Leblanc G \& Rigaud J -L (1998). A new gel-like phase in dodecylmaltoside-lipid mixtures: implications in solubilization and reconstitution studies. Biophysical J ournal, 74: 918-930.

20. Rigaud J $-L$, Paternostre $M T \&$ Bluzat $A$ (1988). Mechanisms of membrane protein insertion into liposomes during reconstitution procedures involving the use of detergents. 2. Incorporation of the lightdriven proton pump bacteriorhodopsin. 
Biochemistry, 27: 2677-2688.

21. Richard P, Rigaud J -L \& Gräber P (1990). Reconstitution of CFOF1 into liposomes using a new reconstitution procedure. European J ournal of Biochemistry, 193: 921-930.

22. Lévy D, Gulik A, Bluzat A \& Rigaud J-L (1992). Reconstitution of the sarcoplasmic reticulum $\mathrm{Ca}^{2+} \mathrm{ATPase}$ : mechanisms of membrane protein insertion into liposomes during reconstitution procedures involving the use of detergents. Biochimica et Biophysica Acta, 1107: 283-298.

23. Rigaud J -L, Lévy $D$, Mosser $G \&$ Lambert O (1998). Detergent removal by BioBeads. Applications to membrane protein reconstitution and 2D crystallization. European Biophysics J ournal, 27: 305-319.

24. Lévy $D$, Bluzat $A$, Seigneuret $M \&$ Rigaud J -L (1990). A systematic study of liposome and proteoliposome reconstitution involving Bio-Bead-mediated Triton X-100 removal. Biochimica et Biophysica Acta, 1025: 179-190.

25. Seigneuret M \& Rigaud J -L (1986). Analysis of passive and light-driven ion movements in large bacteriorhodopsin liposomes reconstituted by reverse-phase evaporation. 2. Influence of passive permeability and back-pressure effects upon light-induced proton uptake. Biochemistry, 25: 6723-6729.

26. Lévy $D$, Seigneuret $M$, Bluzat A \& Rigaud $J$ J (1990). Evidence for proton countertransport by the sarcoplasmic reticulum $\mathrm{Ca}^{2+}$ ATPase during calcium transport in reconstituted proteoliposomes with "low" ionic permeability. J ournal of Biological Chemistry, 265: 19524-19533.

27. Hao L, Rigaud J -L \& Inesi G (1994). $\mathrm{Ca}^{2+}$ / $\mathrm{H}^{+}$countertransport and electrogenicity in proteoliposomes containing erythrocyte plasma membrane, $\mathrm{Ca}^{2+} \mathrm{ATPase}$ and exogenous lipids. Journal of Biological Chemistry, 269: 14268-14275.

28. Salvador J, Inesi G, Rigaud J LL \& Mata AM (1998). $\mathrm{Ca}^{2+}$ transport by reconstituted synaptosomal ATPase is associated with $\mathrm{H}^{+}$countertransport and net charge displacement. J oumal of Biological Chemistry, 273: 18230-18234.

29. Pitard B, Richard P, Dunach M, Girault G \& Rigaud J -L (1996). ATP synthesis by the FOF1 ATP synthase from bacillus PS3 reconstituted into liposomes with bacteriorhodopsin. Factors defining the optimal reconstitution of ATP synthases with bacteriorhodopsin. European J ournal of Biochemistry, 235: 769-778.

30. Pitard B, Richard P \& Rigaud J -L (1996). ATP synthesis by the FOF1 ATP synthase from bacillus PS3 co-reconstituted into liposomes with bacteriorhodopsin. Relationships between proton motive force and ATP synthesis. European J ournal of Biochemistry, 235: 779-788.

31. Richard P, Pitard B \& Rigaud J -L (1995). ATP synthesis by the FOF1 ATPase from the thermophilic bacillus PS3 co-reconstituted with bacteriorhodopsin into liposomes. Evidence for stimulation of ATP synthesis by ATP bound to a non-catalytic site. J ournal of Biological Chemistry, 270: 21571-21578.

32. Steinberg-Yfrach $G$, Rigaud J $L$, Moore LA, Gust D \& Moore TA (1998). Lightdriven, FOF1 ATP synthase-catalyzed synthesis of ATP by an artificial photosynthetic membrane. Nature, 392: 479-482.

33. Lambert $O$, Plançon $L$, Rigaud J $L$ \& Letellier L (1998). Protein-mediated DNA transfer into liposomes. Molecular Microbiology, 30: 761-765.

34. Boehm J, Lambert O, Letellier L, Baumeister W \& Rigaud J -L (2001). FhuAmediated phage genome transfer into liposomes: a cryo-electron tomography study. Current Biology, 11: 1168-1175.

35. Baumeister W, Grimm R \& Walz J (1999). Electron tomography of molecules and cells. Trends in Cell Biology, 9: 81-85.

36. Lambert $O$, Moeck $G$, Lévy $D$, Plançon $L$, Letellier L \& Rigaud J L (1999). A projected structure of FhuA, a ligand-gated channel of $\mathrm{E}$. coli outer membrane protein. J ournal of Structural Biology, 126: 145-155.

37. Lambert $O$, Letellier L, Gelbart WM \& Rigaud J -L (2000). DNA delivery by phage as a strategy for encapsulating toroidal condensates of arbitrary size into liposomes. Proceedings of the National Academy of Sciences, USA, 97: 7248-7253.

38. Ostermeier C, Iwata J \& Michel H (1996). Cytochrome c oxidase. Current Opinion in Structural Biology, 6: 460-466.

39. Nollert $P$, Qiu $H$, Gaffrey $M$, Rosenbusch J P \& Landau EM (2001). Molecular mechanisms for the crystallization of bacteriorhodopsin in lipidic cubic phases. FEBS Letters, 504: 179-186.

40. Rigaud J -L, Mosser G, Lacapère J J, Lévy D, Oloffson A \& Ranck J L (1997). BioBeads: an efficient strategy for 2D crystallization of membrane proteins. J ournal of Structural Biology, 118: 226-235.

41. Mosser G, Breyton C, Oloffson A, Popot J L \& Rigaud J -L (1997). A 8A projection map of cytochrome $b_{6} f$ complex from Chlamydomonas reinhartdii. J ournal of Biological Chemistry, 272: 20263-20268.

42. Lacapère JJ, Stokes $D$, Olofsson $A \&$
Rigaud J -L (1998). Two-dimensional crystallization of $\mathrm{Ca}^{2+}$ ATPase by total detergent removal. Biophysical J ournal, 75: 1319-1329.

43. Saint $N$, Lacapère J J , Ghazi A, Martinac $B$ \& Rigaud J -L (1998). A hexameric transmembrane pore revealed by $2 \mathrm{D}$ crystallization of the large mechanosensitive ion channel (MsCL) of E. coli. J oumal of Biological Chemistry, 273: 14667-14670.

44. Dolder M, Engel A \& Zulauf M (1996). The micelle to vesicle transition of lipids and detergents in the presence of a membrane protein: towards a rationale for $2 \mathrm{D}$ crystallization. FEBS Letters, 382: 203208.

45. Engel A, Hoenger A, Hefti A, Henn C, Ford RC, Kisler J \& Zulauf M (1992). Assembly of 2D membrane protein crystals: dynamics, crystal order and fidelity of structure analysis by electron microscopy. J ournal of Structural Biology, 109: 219234.

46. Cherry RJ , Muller R, Henderson R \& Heyn MP (1997). Temperature-dependent aggregation of bacteriorhodopsin in dipalmitoyl and dimyristoylphosphatidylcholine. J oumal of Molecular Biology, 121: 283298.

47. Young HS, Rigaud J L, Lacapère JJ, Reddy LG \& Stokes DL (1997). How to make tubular crystals by reconstitution of detergent-solubilized Ca-ATPase. Biophysical J ournal, 72: 2545-2558.

48. Mannella C (1984). Phospholipaseinduced crystallization of channels in mitochondrial outer membranes. Science, 224: 165-166.

49. Komberg RD \& Darst SA (1991). Twodimensional crystals of proteins on lipid layers. Current Opinion in Structural Biology, 1: 642-646.

50. Chiu W, Avila-Sakar AJ \& Schmid MF (1997). Electron crystallography of macromolecular periodic arrays on phospholipid monolayers. Advances in Biophysics, 34: 161-172.

51. Lévy D, Mosser G, Lambert O, Moeck G, Bald D \& Rigaud J -L (1999). 2D crystallization on lipid layer: a successful approach for membrane proteins. J ournal of Structural Biology, 127: 44-52.

52. Lévy D, Chami $M \&$ \& Rigaud J -L (2001). Two dimensional crystallization of membrane proteins: the lipid layer strategy. FEBS Letters, 504: 187-193.

53. Dubochet J, Adrian M, Chang JJ, Homo J C, Lepault J , McDonall AW \& Schultz P (1988). Cryo-electron microscopy of vitrified specimen. Quarterly Reviews of Biophysics, 21: 129-228. 
54. Stahlberg H, Fotiadis D, Scheuring $S$, Remigny $\mathrm{H}$, Braun $\mathrm{T}$, Mitsuoka $\mathrm{Y}$, Fujiyoshi $Y \&$ Engel A (2001). Two-dimensional crystals: a powerful approach to assess structure, function and dynamics of membrane proteins. FEBS Letters, 504: 166-172.
55. Scheuring $S$, Reiss Husson $F$, Engel $A$, Rigaud J -L \& Ranck J L (2001). High resolution AFM topographs of Rubrivivax gelatinosus light-harvesting complex $\mathrm{LH} 2$. EMBO J ournal, 20: 3029-3035.

56. Engel $A \&$ Muller DJ (2000). Obsenving single molecules at work with the atomic force microscope. Nature Structural Biology, 7: 715-718.

57. Heymann J B, Müller DJ, Mitsuoka $K \&$ Engel A (1997). Electron and atomic force microscopy of membrane proteins. Current Opinion in Structural Biology, 7: 543549. 